\title{
Conserved Structural Anatomy Between Divergent Viral Capsid Nanoparticles for Vaccine Design
}

\author{
Dustin M. McCraw ${ }^{1}$, Neetu M. Gulati ${ }^{1}$, Mallory L. Myers ${ }^{1}$, Audray K. Harris ${ }^{1}$
}

1. Laboratory of Infectious Diseases, National Institute of Allergy and Infectious Diseases, National Institutes of Health, Bethesda, USA.

Epitopes with valuable characteristics, such as those that elicit broadly-neutralizing antibodies, can be designed to be displayed on the surface of preexisting viral structures. Viral components may also provide valuable characteristics, such as multivalency and high immunogenicity, which may translate to designed vaccine immunogens. By increasing our understanding of natural sequence variations and their link to structural conservations of virus capsids, we can gain additional insights into regions of the viral capsid that can be mutated for the structure-guided design of vaccines. This will facilitate the engineering of epitopes from infectious disease agents onto vaccine scaffolds to produce novel vaccine immunogens [1].

The human hepatitis B virus (HBV) capsid is a nanoparticle platform used in vaccine design strategies. The capsid is a highly immunogenic nanoparticle that contains protruding immunodominant loops that are amendable to insertions [2]. However, little is known about the connection between sequence variation and the structural conservation of HBV capsids from different species. Thus, capsid homologs provide a natural evolutionary system to understand how capsid structure is affected by mutations. In this study, we carried out a comparative analysis between the evolutionary homologs of HBV capsids from three species: (1) Human hepatitis B virus (HBV), (2) Woodchuck Hepatitis B Virus (WHV), and (3) Ground Squirrel Hepatitis B Virus (GSHV). We used the methods of sequence comparison, protein expression and purification, homology modeling, and cryo-electron microscopy (cryo-EM) to compare the sequences and structures of the capsids. We found that the capsids had sequence divergence, but maintained a similar capsid structure, indicating that the capsid is quite tolerate of mutations.

The HBV capsid had $66 \%$ sequence identity to GSHV and WHV, with a significant number of mutations clustered near the immunodominant loop (Figure 1a). Capsids were expressed in E. coli and were purified by gradient centrifugation (Figure 1b). Cryo-EM images of the capsids were acquired on a Titan Krios at $300 \mathrm{keV}$ with a Falcon II direct detector in movie mode to collect multiple frames for each image (Figure 1c-e). For image processing and 3D reconstruction, various software packages were used including CTFFind4 and MotionCor2. Relion 2 was used for 2-D classification and to reconstruct the final 3-D maps. The capsids reconstructed to resolutions of $4.8 \AA$ (HBV) (Figure 2a), $7.9 \AA$ resolution (GSHV) (Figure 2b), and $7.5 \AA$ (WHV) (Figure 2c). A molecular model of HBV (RCSB160181) was fitted to the density map and fitting of secondary structural features such as alpha helices to the map were visible (Figure 2d). Homology models for GSHV and WHV (I-Tasser) were fitted into their respective capsid maps (Figure 2e-f). While at lower resolution, the alpha helical tertiary structure of the homologs were similar to the HBV capsid.

In summary, despite the variation in capsid amino acid sequence, the 3D maps show that the conformation of the immunodominant loops that make up the spikes is generally maintained for all capsids. These results suggest that the viral capsid family is tolerant to mutations in the primary sequence for both the tertiary and quaternary capsid structures. The localization of GSHV and WHV loops in a similar position to the engineerable HBV immunodominant loop suggests that epitope 
insertions would be feasible for each capsid. Thus, the conserved structural anatomy between these sequence divergent viral capsid nanoparticles indicate opportunities for their use as vaccine design scaffolds.

References:

[1] Correia, B.E et al. Nature 507 (2014), p. 201.

[2] Wynne, S. A et al. Mol Cell 3 (6), p. 771.

a

Immunodominant Loop

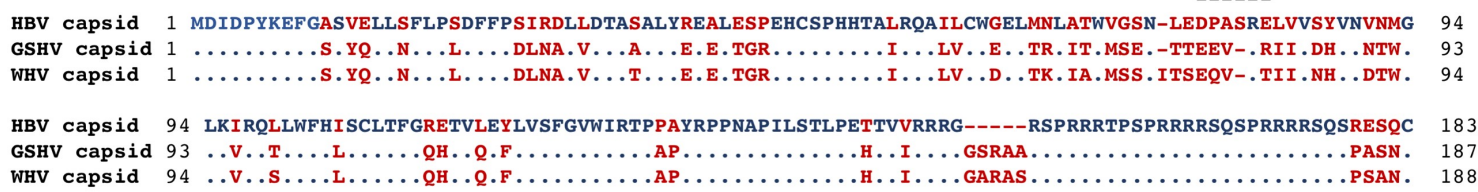
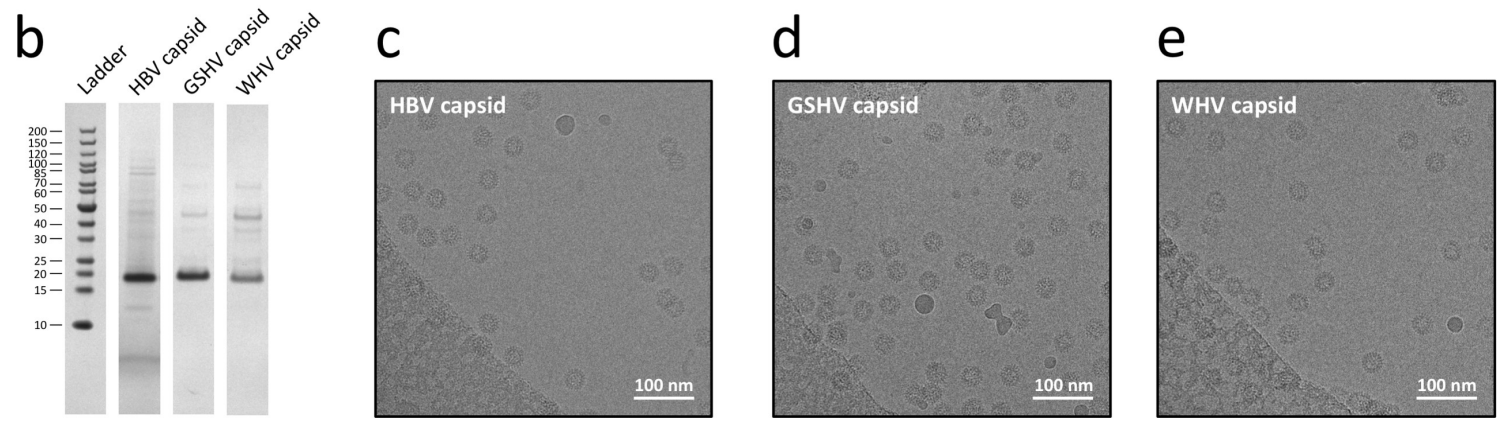

Figure 1. Sequence analysis and preparation of capsids. (a) Sequence alignment of HBV, GSHV, and WHV capsids with conserved amino acids in blue and non-conserved in red. The immunodominant loop is HBV residues 78-83. (b) SDS-PAGE for purified capsids with bands at approx. $20 \mathrm{kDa}$. Cryo-EM images of (c) HBV, (d) GSHV, and (e) WHV capsids showing intact particles. Scale bar $=100 \mathrm{~nm}$.

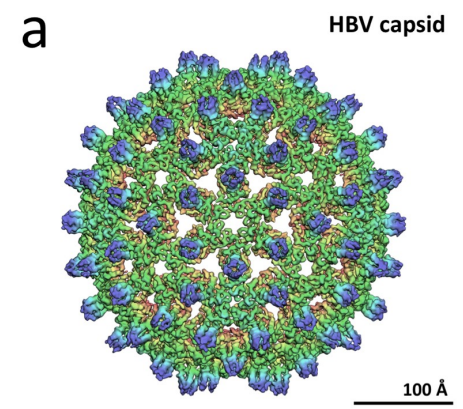

b
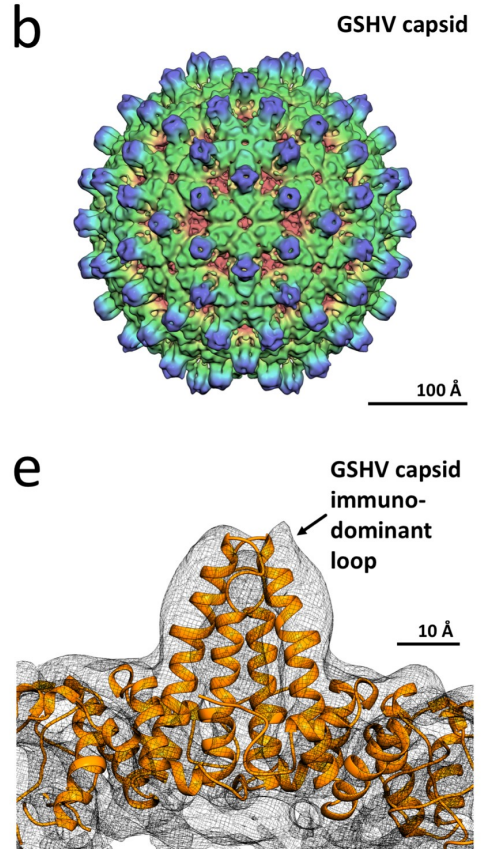

C
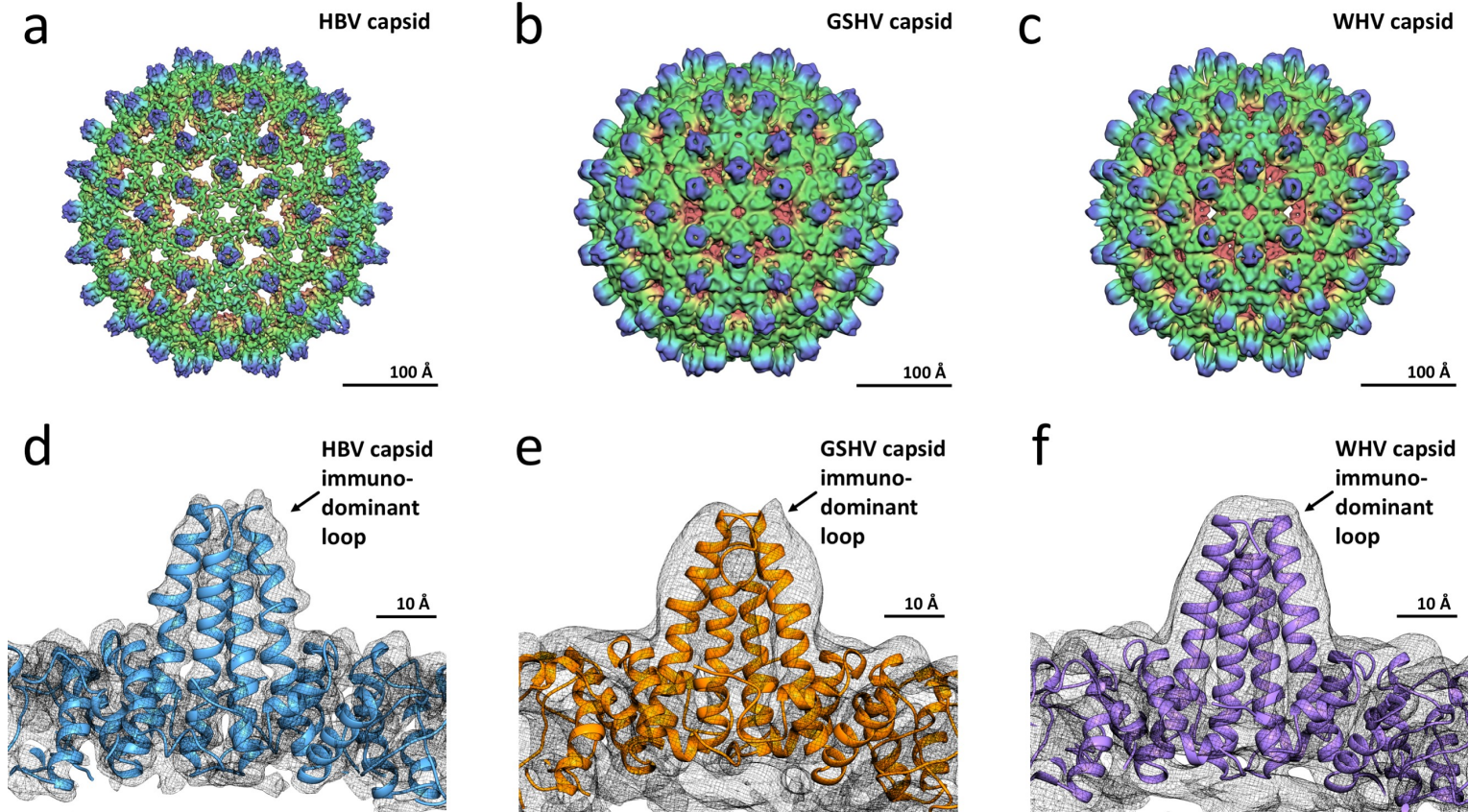

Figure 2. 3D reconstructions of capsids. (a) HBV, (b) GSHV, and (c) WHV capsids with surfaces colored according to radial depth (from red to green to blue). Docked molecular model coordinates into 3D maps for capsids: (d) HBV, (e) GSHV, (f) WHV. Scale bars $100 \AA$, $10 \AA$. 